$$
\begin{gathered}
\text { DOE / IR/403.37-4 } \\
\text { DOE/ER/40537--4 }
\end{gathered}
$$

DE91 005579

\title{
Analysis of Multiparticle Bose-Einstein Correlations in Ultra-Relativistic Heavy Ion Collisions
}

\author{
John G. Cramer \\ Department of Physics \\ University of Washington \\ Seattle WA 98195
}

October 19,1990

\begin{abstract}
We introduce the coalescence va.iables, a set of three boost.invariant kinematic quantities which may be used in analyzing $n$-particle correlations. These variabies characterize the invariant mass of an $n$-particle system and in three directions and separate the timelike and spacelike characteristics of the source. The analytic Kolehmanien-Gyulassy model is generalized to give two, three, and four-particle correlation functions, with coherence and Coulomb corrections applied to the basic formalism. We demonstrate the relation of the coalescence variables to the radius and duration of the source, and find that for sufficiently large transverse radii, Coulomb cffects can suppress the structure of the Hanbury-Brown-Twiss correlations so that no significant information on source size can be obtained.
\end{abstract}

PACS numbers: 05.30.J,12.38.M;07.60.L 


\section{DISCLAIMER}

This report was prepared as an account of work sponsored by an agency of the United States Government. Neither the United States Government nor any agency thereof, nor any of their employees, makes any warranty, express or implied, or assumes any legal liability or responsibility for the accuracy, completeness, or usefulness of any information, apparatus, product, or process disclosed, or represents that its use would not infringe privately owned rights. Reference herein to any specific commercial product, process, or service by trade name, trademark, manufacturer, or otherwise does not necessarily constitute or imply its endorsement, recommendation, or favoring by the United States Government or any agency thereof. The views and opinions of authors expressed herein do not necessarily state or reflect those of the Urited States Government or any agency thereof. 


\section{INTRODUCTION}

In ultra-relativistic collisions between heavy ions, the number of like-sign pions produced in a single collision is expected to be on the order of $10^{3}$. Because of this very large multiplicity, Hanbury-Brown-Twiss correlations between pions may offer a powerful probe for the investigation of such collisions on an event-by-event basis. The numerical factors implicit in multi-particle Bose-Einstein interferometry imply a strong tendency for pions to cluster or "coalesce" in the same region of momentum space due to their mutual Bose-Einstein reinforcement. Therefore, intereferometry using pions clustered in momentum space may offer an important analysis tool. Indeed, it has been suggested ${ }^{1,2}$ that pion "speckle interferometry", i.e., the high-order correlations of pion clusters or "speckles", might be used to extract detailed information on the size, shape, time duration, and eccentricity of the source of pion emission.

A fundamental problem encountered in interferometry using correlations between $n$ particles, where $n$ is larger than 2 , is finding a compact set of independent Lorentz-invariant kinematic basis variables for presenting and analyzing the correlations. The relative vector momenta of $n$ particles require $3^{n-1}$ independent variables for complete specification. This number of parameters is far too large and too inter-related for meaningful analysis. For example, analysis using the relative vector momenta in the correlation of a five-pion system would require 27 independent momentum variables.

Goldhaber ${ }^{3}$ was able to compare the correlations of two and three pion systems by plotting both correlation distributions against a variable which he called $Q^{2}$, the mean square deviation of the invariant mass of the $n$-pion system from its minimum possible value, i.e., $Q^{2}=E^{\mu} E_{\mu}-\left(n \mu_{\pi}\right)^{2}$. Liu, et al. ${ }^{4}$ have employed this $Q^{2}$ variable in analyzing two and three pion correlations in $\mathrm{Ar}+\mathrm{Pb}$ and $\mathrm{Ar}+\mathrm{KCl}$ heavy ion collisions. For the purposes of the present discussion, we will refer to $Q$ as the overall 
coalescence variable, since it goes to zero when a system of pions has coalesced to occupy a minimum volume of momentum space. The use of $Q^{2}$ and similar variables in the analysis of correlations of relativistic particles has been criticized $^{5,6}$ because, although it is a Lorentz-invariant quantity, it mixes the time-like and space-like characteristics of the source. In central collisions of ultra-relativistic heavy ions the time and space (or longitudinal and transverse) source characteristics provide independent information about the collision, and is important to keep these separated in the analysis of multiparticle correlations.

The invariant mass of a two pion system can exceed the minimum value of $2 \mu_{\pi}$ only if the two pions have non-zero relative momentum. Consider the distribution of emitted pions in a spherical coordinate system $(r, \theta, \phi)$ where the beam direction is the $z$ or longitudinal axis defined by $\theta=\pi / 2$ and the locus $\theta=0$ is the transverse (equatorial) plane. Thus, $\theta$ has the range $-\frac{\pi}{2} \leq \theta \leq \frac{\pi}{2}$. The relative momentum between a pair of emitted pions can have longitudinal $(\theta)$, transverse $(\phi)$, and radial ( $r$ ) components. In the context of Hanbury-Brown-Twiss correlations, these three momentum differences sample separate geometrical aspects of the pion source and should, if possible, be investigated separately. In what follows, we propose a decomposition of the Goldhaber coalescence variable into longitudinal, transverse, and radial coalescence components, thereby preserving this distinction. We then apply this analysis technique to a comparison of two, three, and four pion systems using a generalization of the analytic model of Kolehmainen and Gyulassy ${ }^{7,8}$.

\section{COALESCENCE IN THE 2-PARTICLE SYSTEM}

We will use the following notation. The rest mass of the $i$ th particle is $\mu_{\pi}, i$. e. the pion mass. Its total energy is $w_{i}$, its vector momentum is $\vec{P}_{i}$, its transverse momentum is $p_{i}$, its transverse mass is $m_{i}=\sqrt{p_{i}^{2}+\mu_{\pi}^{2}}$, its longitudinal momentum 
is $q_{i}=\sqrt{w_{i}^{2}-m_{i}^{2}}$, its azimuthal angle in the transverse plane is $\phi_{i}$, its polar angle forward or backward of the transverse plane is $\theta_{i}$, and its rapidity is $y_{i}=\frac{1}{2} \log \left[\left(w_{i}+\right.\right.$ $\left.\left.q_{i}\right) /\left(w_{i}-q_{i}\right)\right] \approx \log \left[\left(1+\sin \theta_{i}\right) / \cos \theta_{i}\right]$. We will also employ the useful rapidity relations $w_{i}=m_{i} \cosh \left(y_{i}\right)$ and $q_{i}=m_{i} \sinh \left(y_{i}\right)$. We will take $c=1$ and express all of the ahove quantities except angle and rapidity in energy units, usually $\mathrm{MeV}$.

For a two particle system, the Goldhaber coalescence variable is given by:

$$
\begin{aligned}
Q_{2}^{2}= & \left(w_{1}+w_{2}\right)^{2}-\left(\vec{P}_{1}+\vec{P}_{2}\right)^{2}-\left(2 \mu_{\pi}\right)^{2} \\
= & \left(w_{1}+w_{2}\right)^{2}-\left(p_{1} \cos \phi_{1}+p_{2} \cos \phi_{2}\right)^{2} \\
& -\left(p_{1} \sin \phi_{1}+p_{2} \sin \phi_{2}\right)^{2}-\left(q_{1}+q_{2}\right)^{2}-\left(2 \mu_{\pi}\right)^{2} .
\end{aligned}
$$

With suitable algebraic manipulation, this can be reduced to the form:

$$
Q_{2}^{2}=2\left\{m_{1} m_{2}\left[1+2 \sinh ^{2}\left(\frac{y_{1}-y_{2}}{2}\right)\right]-p_{1} p_{2}\left[1-2 \sin ^{2}\left(\frac{\phi_{1}-\phi_{2}}{2}\right)\right]-\mu_{x}^{2}\right\}
$$

We make the following definitions of dimensionless coalescence variables:

$$
\begin{aligned}
C_{2 L}(1,2) & =\frac{1}{\mu_{\pi}} \sqrt{2 m_{1} m_{2}} \sinh \left|\frac{y_{1}-y_{2}}{2}\right|, \\
C_{2 T}(1,2) & =\frac{1}{\mu_{\pi}} \sqrt{2 p_{1} p_{2}} \sin \left|\frac{\phi_{1}-\phi_{2}}{2}\right|, \text { and } \\
C_{2 R}(1,2) & =\frac{1}{\mu_{\pi}} \sqrt{m_{1} m_{2}-p_{1} p_{2}-\mu_{\pi}^{2}} \\
& =\frac{1}{\mu_{\pi}} \sqrt{\left(m_{1}-m_{2}\right)^{2}-\left(p_{1}-p_{\check{\iota}}\right)^{2}} \\
& =\frac{1}{\mu_{\pi}} \sqrt{\left(m_{1}+m_{2}\right)^{2}-\left(p_{1}+p_{2}\right)^{2}-\left(2 \mu_{\pi}\right)^{2}} .
\end{aligned}
$$

Here $C_{2 L}$ is the two particle longitudinal coalescence, $C_{2 T}$ is the two particle transverse coalescence, and $C_{2 R}$ is the two particle radial coalescence. They are dimensionless variables which specify the invariant mass deviation relative to $\mu_{\pi}$, the pion mass. 
Note that $C_{2 L}=0$ when $y_{1}=y_{2}$, that $C_{2 T}=0$ when $\phi_{1}=\phi_{2}$, and that $C_{2 R}=0$ when either $m_{1}=m_{2}$ or $p_{1}=p_{2}$ (since each equality implies the other). The overall two particle coalescence $Q_{2}$ is given by the relation $Q_{2}^{2}=2 \mu_{\pi}^{2}\left(C_{2 L}^{2}+C_{2 T}^{2}+C_{2 R}^{2}\right)$, i.e., the three coalescence components add in quadrature, as would be expected of orthogonal coordinates.

\section{COALESCENCE IN THE N-PARTICLE SYSTEM}

Now let us consider the case of $n$ correlated particles, where $n>2$. The $n$ particle coalescence is given by

$$
Q_{n}^{2}=\left(\sum_{i=1}^{n} w_{i}\right)^{2}-\left(\sum_{i=1}^{n} \vec{P}_{i}\right)^{2}-\left(\sum_{i=1}^{n} \mu_{\pi}\right)^{2}
$$

which with suitable manipulation becomes

$$
Q_{n}^{2}=\sum_{i \neq j}^{n} m_{i} m_{j}\left[1+2 \sinh ^{2}\left(\frac{y_{i}-y_{j}}{2}\right)\right]-\sum_{i \neq j}^{n} p_{i} p_{j}\left[1-2 \sin ^{2}\left(\frac{\phi_{i}-\phi_{j}}{2}\right)\right]-\sum_{i \neq j}^{n} \mu_{\pi}^{2} .
$$

Thus, using definitions (3), (4), and (5),

$$
\begin{aligned}
& \qquad \begin{aligned}
Q_{n}^{2} & =2 \mu_{\pi}^{2} \sum_{i<j}^{n}\left[C_{2 L}^{2}(i, j)+C_{2 T}^{2}(i, j)+C_{2 R}^{2}(i, j)\right] \\
& =2 \mu_{\pi}^{2}\left(C_{n L}^{2}+C_{n T}^{2}+C_{n R}^{2}\right),
\end{aligned} \\
& \text { where } C_{n X}^{2}=\sum_{i<j}^{n} C_{2 X}^{2}(i, j) \text { with } X=L, T \text {, or } R \text {. }
\end{aligned}
$$

These generalized coalescence variables can be calculated for a system composed of any number of pions, given the momentum components of each particle of the system. The coalescence variables for all particle numbers have the same significance, denoting the amount by which the momentum mismatch along a particular axis increases the invariant mass of the system, in units of $\mu_{\pi}$. Thus, as we will see, coalescence variables can be useful in comparing $n$-particle correlations over a range of values of $n$. 
For the purposes of the comparisons presented in this paper we will use a simple linear parameterization of the mornentum variables in terms of the kinematic parameters $y_{0}, \delta y, m_{0}, \delta m, \phi_{0}$, and $\delta \phi$, the central value and difference of the rapidity, transverse mass, and azimuthal angle, respectively. For the two particle case, we will take $y_{1}=y_{0}+\delta y, y_{2}=y_{0}-\delta y, m_{1}=m_{0}+\delta m, m_{2}=m_{0}-\delta m, \phi_{1}=\phi_{0}+\delta \phi$, and $\phi_{2}=\phi_{0}-\delta \phi$. Thus $m_{0}=\left(m_{1}+m_{2}\right) / 2$ and $\delta m=\left(m_{1}-m_{2}\right) / 2$, etc. For the three particle case, we will take $y_{1}=y_{0}+\delta y^{\prime}, y_{2}=y_{0}, y_{3}=y_{0}-\delta y^{\prime}, m_{1}=m_{0}+\delta m^{\prime}, m_{2}=m_{0}, m_{3}=m_{0}-\delta m^{\prime}$, $\phi_{1}=\phi_{0}+\delta \phi^{\prime}, \phi_{2}=\phi_{0}$, and $\phi_{3}=\phi_{0}-\delta \phi^{\prime}$, where $\delta y^{\prime}=0.86 \times \delta y, \delta m^{\prime}=0.86 \times \delta m$, and $\delta \phi^{\prime}=0.86 \times \delta \phi$. For the four particle case, we will take $y_{1}=y_{0}+\delta y^{\prime \prime}, y_{2}=y_{0}+\delta y^{\prime \prime} / 3$, $y_{3}=y_{0}-\delta y^{\prime \prime} / 3, y_{4}=y_{0}-\delta y^{\prime \prime}, m_{1}=m_{0}+\delta m^{\prime \prime}, m_{2}=m_{0}+\delta m^{\prime \prime} / 3, m_{3}=m_{0}-\delta m^{\prime \prime} / 3$, $m_{4}=m_{0}-\delta m^{\prime \prime}, \phi_{1}=\phi_{0}+\delta \phi^{\prime \prime}, \phi_{2}=\phi_{0}+\delta \phi^{\prime \prime} / 3, \phi_{3}=\phi_{0}-\delta \phi^{\prime \prime} / 3$, and $\phi_{4}=\phi_{0}-\delta \phi^{\prime \prime}$, where $\delta y^{\prime \prime}=0.72 \times \delta y, \delta m^{\prime \prime}=0.72 \times \delta m$, and $\delta \phi^{\prime \prime}=0.72 \times \delta \phi$.

Reduction factors have been applied to the $n=3$ and $n=4$ difference parameters above give the corresponding coalescence variables about the same dependence as the $\mathrm{n}=2$ coalescence variables. Fig. 1 shows plots of the coalescence variables $C_{n L}, C_{n T}$, and $C_{n R}$ plotted against the difference parameters $\delta \mathrm{y}, \delta \phi$, and $\delta \mathrm{m}$ for $y_{0}=\phi_{0}=0, \mathrm{n}=2$, 3 , and 4 , and $m_{0}=3 \mu_{\pi}$. We see that all three coalescence variables are single valued monotonically increasing functions of $\delta y, \delta \phi$, and $\delta m$ and that $C_{2 X}, C_{3 X}$, and $C_{4 X}$ ( $X=L, T$ and $R$ ) have similar behavior and normalizations.

\section{APPLICATION TO 2, 3, AND 4-PARTICLE CORRELATIONS}

Up to now, investigations of the correlations of pions produced in ultrarelativistic heavy ion collisions have focused on two particle systems, with a few studies of three particle correlations. However, we expect that with the higher multiplicities expected collisions at RHIC and LHC energies, correlations with larger numbers of pions (and kaons) will play a prominent role in the analysis. Here we will 
use the coalescence variables to compare the correlations of two to four pions.

We are particularly interested in the competition between the rising strength due to Bose-Einstein attraction exhibited by the general $n$-particle correlation $R_{n}$, which for neutral particles has a maximum value of $n$ ! when the overall coalescence $Q^{2}=0$, and the increasing suppression of the charged pion correlation $R_{n}^{[t]}$ due to Coulomb repulsion, which increases approximately as $F\left(\eta_{\text {average }}\right)^{n(n-1) / 2}$ due to the mutual repulsion of charged pions which are closely correlated. This competition ultimately depends on formidable and unresolved theoretical issues, particularly the derivation of a reliable expression for multiparticle Coulomb effects. In the present work the Bose-Einstein/Coulomb competition will be investigated through the use of an analytic model for the correlations and a simple but plausible Coulomb correction procedure.

Kolehmainen and Gyulass $y^{7,8}$ have presented a boost-invariant analytic model for predicting the one and two particle correlations of pions from an ultra-relativistic heavy ion collision. The model is able to obtain an analytic expression for the correlation distribution by employing a simple pseudo-thermal description of the pion momentum distribution function. It uses the inside-outside cascade model ${ }^{9}$ to describe the pion emission process and assumes that the emissions are completely incoherent. It characterizes the pion-emitting volume with three parameters, the transverse radius $r_{\Gamma}$ of the emitting volume, and the time constant $\tau_{0}$ of the emission process, and the pseudo-temperature $T$ of the source. The pseudo-temperature of the Kolehmainen and Gyulassy model is related to the physical temperature $U$ of a thermal model by the relation $T=1.42 \times U-12.7 \mathrm{MeV}$. In the calculations below, we will usually take $U=\mu_{\pi}$ which is equivalent to $T=186.1 \mathrm{MeV}$.

The one particle inclusive pion yield given by this model is: 


$$
\frac{d^{3} N_{\pi}}{d^{3} P_{1}}=A K_{0}\left(m_{1} / T\right)
$$

where $A$ is a normalization factor and $K_{0}(z)$ is a modified irregular cylindrical Bessel function of order zero with possibly complex argument $z$. We will also consider the $n$-particle correlation distributions, here defined as the ratio of the $n$-particle inclusive pion yield to the product of $n$ one-particle inclusive pion yields, i.e.,

$$
R_{n}\left(\vec{P}_{1}, \cdots \vec{P}_{n}\right)=\frac{d^{3 n} N_{\pi}}{d^{3} P_{1} \cdots d^{3} P_{n}} / \prod_{i=1}^{n} \frac{d^{3} N_{\pi}}{d^{3} P_{i}}
$$

The two particle correlation distribution for neutral particles (superscript [0]), in the Kolehmainen-Gyulassy model, is given by:

$$
R_{2}^{[0]}\left(\vec{P}_{1}, \vec{P}_{2}\right)=1+B_{12}^{2}
$$

where

$$
\begin{gathered}
B_{12}=\frac{\left|G\left(\vec{P}_{1}, \vec{P}_{2}\right)\right|}{\sqrt{G\left(\vec{P}_{1}, \vec{P}_{1}\right) G\left(\vec{P}_{2}, \vec{P}_{2}\right)}}, \text { and } \\
G\left(\vec{P}_{1}, \vec{P}_{2}\right)=A K_{0}\left(\sqrt{u_{12}}\right) \exp \left[-\Delta p_{12}^{2}\left(\frac{r_{T}}{2 \hbar}\right)^{2}\right], \text { with } \\
\Delta p_{12}^{2}=p_{1}^{2}+p_{2}^{2}-2 p_{1} p_{2} \cos \left(\phi_{1}-\phi_{2}\right), \text { and } \\
u_{12}=\left[\frac{m_{1}+m_{2}}{2 T}+\frac{i \tau_{0}}{\hbar}\left(m_{1}-m_{2}\right)\right]^{2} \\
+4\left[\left(\frac{1}{2 T}\right)^{2}+\left(\frac{\tau_{0}}{\hbar}\right)^{2}\right] m_{1} m_{2} \sinh ^{2}\left|\frac{y_{1}-y_{2}}{2}\right|
\end{gathered}
$$

In terms of the coalescence variables we can write (16) and (17) as

$$
\begin{gathered}
\Delta p_{12}^{2}=\left(m_{1}+m_{2}\right)^{2}+2 \mu_{\pi}^{2}\left(C_{2 R}^{2}+C_{2 T}^{2}\right), \text { and } \\
u_{12}=\left[\frac{m_{1}+m_{2}}{2 T}+\frac{i \tau_{0}}{\hbar}\left(m_{1}-m_{2}\right)\right]^{2}+2\left[\left(\frac{1}{2 T}\right)^{2}+\left(\frac{\tau_{0}}{\hbar}\right)^{2}\right] \mu_{\pi}^{2} C_{2 L}^{2} .
\end{gathered}
$$


Note that $u_{12}$ is a complex quantity which becomes purely real when $\left(m_{1}-m_{2}\right)=0$. The quantity $\left(m_{1}-m_{2}\right)$ in equation $(19)$ is closely related to the radial coalescence $C_{2 R}$, but no simple function of the latter can be used in the equation.

In the present work we have generalized the Kolehmainen-Gyulassy model summarized in equations (11) through (19) in several ways. First, following the work of Biyajima, et al. ${ }^{11}$, we have used quantum optics (QO) interference diagrams to derive the two, three, and four particle correlation distributions for neutral particles in terms of the Kolehmainen-Gyulassy model with the addition of coherence. The Biyajima formalism includes the possibility of a coherent contribution to particle emission, but implicitly assumes that there is only one source of coherent emission. The two particle correlation function, calculated in this way, is a generalization of equation (13) and has the form:

$$
R_{2}^{[0]}\left(\vec{P}_{1}, \vec{P}_{2}\right)=1+\epsilon^{2} B_{12}^{2}+2 \epsilon(1-\epsilon) B_{12}
$$

Here the parameter $\epsilon$ specifies the fraction of the net emission of the source that is incoherent and is defined as $\epsilon=m_{\text {incoherent }} /\left(m_{\text {incoherent }}+m_{\text {coherent }}\right)$, where $m_{x}$ is the pion multiplicity of type $x$. When coherent emission of pions is significant, $\epsilon<1$ and this has the effect of reducing the peaking near $Q^{2}=0$ of the correlation distributions. In the calculated examples presented later in this paper we will assume that the pion source is completely incoherent, i.e., $\epsilon=1$.

Th': three neutral particle correlation derived from QO diagrams is:

$$
\begin{aligned}
R_{3}^{[0]}\left(\vec{P}_{1}, \vec{P}_{2}, \vec{P}_{3}\right)=1 & +\epsilon^{2}\left(B_{12}^{2}+B_{23}^{2}+B_{31}^{2}\right) \\
& +2 \epsilon^{3}\left(B_{12} B_{23} B_{31}\right) \\
& +2 \epsilon(1-\epsilon)\left(B_{12}+B_{23}+B_{31}\right) \\
& +2 \epsilon^{2}(1-\epsilon)\left(B_{12} B_{3}+B_{13} B_{32}+B_{21} B_{13}\right)
\end{aligned}
$$


and the four neutral particle correlation is:

$$
\begin{aligned}
R_{4}^{[0]}\left(\vec{P}_{1}, \vec{P}_{2}, \vec{P}_{3}, \vec{P}_{4}\right)=1 & +\epsilon^{2}\left(B_{12}^{2}+B_{13}^{2}+B_{14}^{2}+B_{23}^{2}+B_{24}^{2}+B_{34}^{2}\right) \\
& +2 \epsilon^{3}\left(B_{12} B_{23} B_{31}+B_{12} B_{24} B_{41}+B_{13} B_{34} B_{41}+B_{23} B_{34} B_{42}\right) \\
& +2 \epsilon^{4}\left(B_{12} B_{23} B_{34} B_{41}+B_{12} B_{24} B_{43} B_{31}+B_{13} B_{32} B_{24} B_{41}\right) \\
& +\epsilon^{4}\left(B_{12}^{2} B_{34}^{2}+B_{13}^{2} B_{24}^{2}+B_{14}^{2} B_{23}^{2}\right) \\
& +2 \epsilon(1-\epsilon)\left(B_{12}+B_{13}+B_{14}+B_{23}+B_{24}+B_{34}\right) \\
& +3 \epsilon^{2}(1-\epsilon)\left(B_{12} B_{23}+B_{12} B_{24}+B_{13} B_{34}+B_{23} B_{34}\right. \\
& \left.+B_{12} B_{31}+B_{12} B_{41}+B_{13} B_{41}+B_{23} B_{42}\right) \\
& +4 \epsilon^{2}(1-\epsilon)^{2}\left(B_{12} B_{34}+B_{13} B_{24}+B_{14} B_{23}\right) \\
& +2 \epsilon^{3}(1-\epsilon)\left(B_{12} B_{23} B_{34}+B_{12} B_{24} B_{43}+B_{13} B_{32} B_{24}\right. \\
& +B_{12} B_{23} B_{41}+B_{12} B_{24} B_{31}+B_{13} B_{32} B_{41} \\
& +B_{12} B_{34} B_{41}+B_{12} B_{43} B_{31}+B_{13} B_{24} B_{41} \\
& \left.+B_{23} B_{34} B_{41}+B_{24} B_{43} B_{31}+B_{32} B_{24} B_{41}\right) \\
& +2 \epsilon^{3}(1-\epsilon)\left(B_{12} B_{34}^{2}+B_{13} B_{24}^{2}+B_{14} B_{23}^{2}\right. \\
& \left.+B_{12}^{2} B_{34}+B_{13}^{2} B_{24}+B_{14}^{2} B_{23}\right) \\
&
\end{aligned}
$$

Note that for completely incoherent systems, the $(1-\epsilon)$ terms in the above relations will vanish, resulting in considerable simplification of the equations.

As a second generalization of the Kolehmainen-Gyulassy model, we have included an approximate correction for the mutual Coulomb repulsion of the emitted identical particles. This allows us to obtain $R_{n}^{[ \pm j}\left(\vec{P}_{1}, \cdots \vec{P}_{n}\right)$, the $n$ particle correlation distributions for charged particles (superscript $[ \pm]$ ). Following Gyulassy, et al. ${ }^{10}$, we have used for two charged particle correlations a Coulomb correction of the form:

$$
R_{2}^{[ \pm]}\left(\vec{P}_{1}, \vec{P}_{2}\right)=F\left(\eta_{12}\right) R_{2}^{[0]}\left(\vec{P}_{1}, \vec{P}_{2}\right)
$$


where $F\left(\eta_{12}\right)$ is the Gamow penetrability function $2 \pi \eta_{12} /\left[\exp \left(2 \pi \eta_{12}\right)-1\right]$ and $\eta_{12}$ is the Sommerfeld parameter $\alpha / \beta_{12}$. Here $\alpha$ is the fine structure constant and $\beta_{12}$ is the velocity of particle 1 relative to particle 2 divided by the velocity of light. For the case of pions with kinetic energies comparable to $\mu_{\pi}$, the quantity $2 \pi \eta$ in the penetrability function is typically much less than 1 , which implies that an expansion of the Coulomb correction in powers of $2 \pi \eta$ should converge rapidly. As a correction for final state interaction effects, the use of the Gamow penetrability is a valid approximation only when non-Coulomb final state interactions can be neglected and when the Bohr radius of the system of two charged particles is large compared to the dimensions of the emission source. For pions, the two particle distance scale implied by the Bohr radius is about $193 \mathrm{fm}$, a large distance compared to expected source sizes of 4 to $40 \mathrm{fm}$.

Liu, et $a l^{4}$, have suggested an ad hoc product-form Coulomb correction for a system of three charged particles of the form $\left[F\left(\eta_{12}\right) F\left(\eta_{23}\right) F\left(\eta_{31}\right)\right]$. Here $F\left(\eta_{i j}\right)$ is the Gamow penetrability, and $\eta_{i j}$ the Sommerfeld parameter describing the Coulomb interaction of particles $i$ and $j$. We can provide some justification for a correction of this form by considering the following gedankenexperiment: let us "assemble" an $n$-pion system by bringing the pions one at a time from infinite relative momentum down to the momentum state appropriate to the $\eta$ value of the new pion with respect to the already assembled pions. In this case the net penetrability of the assembled system, and therefore the suppression of the correlation, will be of the form $[F(\bar{\eta}) F(2 \bar{\eta}) F(3 \bar{\eta}) \cdots F((n-1) \bar{\eta})]$, where $\bar{\eta}$ is some average Sommerfeld parameter of the various pion pairs. For two charged pions, this is just the Coulomb correction given in equation (23). For three charged pions, thigi leads to a Coulomb correction of the form $[F(\bar{\eta}) F(2 \bar{\eta})]$. To order $\eta^{2}$, this can be approximated by $[F(\bar{\eta})]^{3}$, essentially the correction suggested by Liu, et al. ${ }^{4}$. However, a better approximation that is valid to order $\eta^{7}$ can be obtained by introducing $\eta^{\prime}$, the $\mathrm{n}=3$ effective Sommerfeld parame- 
ter, which is defined by the relation $F(\eta) F(2 \eta) /\left[F\left(\eta^{\prime}\right)\right]^{3}=1+\mathcal{O}\left(\eta^{7}\right)$. We use the series expansion $x^{\prime}=x+(1 / 9) x^{2}-(1 / 27) x^{3}-(19 / 1215) x^{4}+(11 / 1215) x^{5}+(967 / 229635) x^{6}$ where $x=2 \pi \eta$ and $x^{\prime}=2 \pi \eta^{\prime}$. The Coulomb, correction can then be symmetrized in the particle indices to give a co ction valid to order $\eta^{7}$ that has the form $\left[F\left(\eta_{12}^{\prime}\right) F(\cdot, 23) F\left(\eta_{31}^{\prime}\right)\right]$. The three particle correlation function for charged particles is then:

$$
R_{3}^{[ \pm]}\left(\vec{P}_{1}, \vec{P}_{2}, \vec{P}_{3}\right)=\left[F\left(\eta_{12}^{\prime}\right) F\left(\eta_{23}^{\prime}\right) F\left(\eta_{31}^{\prime}\right)\right] R_{3}^{[\mathrm{c}]}\left(\vec{P}_{1}, \vec{P}_{2}, \vec{P}_{3}\right)
$$

where $\eta_{i j}^{\prime}$ is the $n=3$ effeciive Sommerfeld pararneter defined above.

This conceptual approach to Coulomb corrections can be extended to a system of four charged particles, where it has the form $F(\bar{\eta}) F(2 \bar{\eta}) F(3 \bar{\eta})$. To order $\eta^{\text {? }}$, this can be approximated by $[F(\bar{\eta})]^{6}$, but a better approximation valid to order $\eta^{7}$ can be obtained by introducing $\eta^{\prime \prime}$, the $n=4$ effective Sommerfeld parameter, which is defined by the relation $F(\eta) F(2 \eta) F(3 \eta) /\left[F\left(\eta^{\prime \prime}\right)\right]^{6}=1+\mathcal{O}\left(\eta^{7}\right)$. We use the series expansion $x^{\prime \prime}=x+(2 / 9) x^{2}-(2 / 27) x^{3}-(167 / 2430) x^{4}+(1 / 30) x^{5}+(18091 / 459270) x^{6}$ where $x=2 \pi \eta$ and $x^{\prime \prime}=2 \pi \eta^{\prime \prime}$. The Coulomb correction can then be symmetrized in the particle indices to give a correction valid to order $\eta^{7}$ of the form: $\left[F\left(\eta_{12}^{\prime \prime}\right) F\left(\eta_{13}^{\prime \prime}\right) F\left(\eta_{14}^{\prime \prime}\right) F\left(\eta_{23}^{\prime \prime}\right) F\left(\eta_{24}^{\prime \prime}\right) F\left(\eta_{34}^{\prime \prime}\right)\right]$, where $\eta_{i j}^{\prime \prime}$ is the $\mathrm{n}=4$ effective Sommerfeld parameter defined above. The four particle correlation for charged particles is then:

$$
\begin{aligned}
R_{4}^{[ \pm]}\left(\vec{P}_{1}, \vec{P}_{2}, \vec{P}_{3}, \vec{P}_{4}\right)= & {\left[F\left(\eta_{12}^{\prime \prime}\right) F\left(\eta_{13}^{\prime \prime}\right) F\left(\eta_{14}^{\prime \prime}\right) F\left(\eta_{23}^{\prime \prime}\right) F\left(\eta_{24}^{\prime \prime}\right) F\left(\eta_{34}^{\prime \prime}\right)\right] } \\
& \times R_{4}^{[0]}\left(\vec{P}_{1}, \vec{P}_{2}, \vec{P}_{3}, \vec{P}_{4}\right)
\end{aligned}
$$

The Coulomb corrections of equations (24) and (25) are based on a somewhat schematic conceptual model, the use of which is justified only because a more accurate multi-particle Coulomb correction is not available. We note, however, that both of these Coulomb corrections exhibit an important and required property: when 
one of $n$ correlated particles $x$ is made electrically neutral or given a large relative velocity (so that all of its penetrability factors $F\left(\eta_{i x}\right) \rightarrow 1$ ), the Coulomb correction reduces to that appropriate to the next lowest order correlation, i.e., the correction for $n-1$ particles.

Fig. 2 shows a comparison of neutral and charged 2, 3, and 4 particle correlation distributions $R_{n}^{[ \pm, 0]}$ as functions of the longitudinal coalescence. The fixed parameters for this plot are $y_{0}=\phi_{0}=\delta m=\delta \phi=0, \epsilon=1, m_{0}=3 \mu_{\pi}, r_{T}=1.0 \mathrm{fm}, \tau_{0}=2.0 \mathrm{fm} / \mathrm{c}$, and $\mathrm{T}=185.5 \mathrm{MeV}$. The longitudinal coalescence was varied over $\mathrm{C} \leq C_{n L} \leq 4$. The dashed lines show the neutral particle correlation and the solid lines show the charged particle correlations. The effect of the Coulomb torce in suppressing the correlation at small relative momenta is apparent in these curves, as is the greater enhancement over uncorrelated background $\left(S_{n}=1\right)$ of the $n$ particle correlation distribution.

Fig. 3 shows a comparison of neutral and charged 2, 3, and 4-particle correlation distributions $R_{n}^{[ \pm, 0]}$ as functions of the transverse coalescence. The fixed parameters for these calculated functions are $y_{0}=\delta y=\phi_{0}:=\delta m=0, \epsilon=1, m_{0}=3 \mu_{\pi}$, $r_{T}=1.0 \mathrm{fm}, \tau_{0}=2.0 \mathrm{fm} / \mathrm{c}$, and $\mathrm{T}=185.5 \mathrm{MeV}$. The transverse coalescence was varied over $0 \leq C_{n T} \leq 4$.

Fig. 4 shows a similar comparison of neutral and charged 2, 3, and 4particle correlation distributions $R_{n}^{[ \pm, 0]}$ as functions of the radial coalescence. The fixed parameter $s$ for these calculated functions are $y_{0}=\delta y=\phi_{0}=\delta \phi=0, \epsilon=1, m_{0}=3 \mu_{\pi}$, $r_{T}=1.0 \mathrm{fm}, \tau_{0}=2.0 \mathrm{fm} / \mathrm{c}$, and $\mathrm{T}=185.5 \mathrm{MeV}$. The radial coalescence was varied over $0 \leq C_{n R} \leq 1.4$

\section{DEPENDENCE ON TRANSVERSE RADIUS $\boldsymbol{R}_{T}$}

Equations (15) and (18) imply that the function $G\left(\vec{P}_{1}, \vec{P}_{2}\right)$ has a term which is a Gaussian exponential with an exponent of the form $-\frac{1}{2}\left[\left(\mu_{\pi} / \hbar\right) r_{T} C_{2 T}\right]^{2}$ and $a$ 
similar term involving $C_{2 R}$. This means that the transverse has a complementary relationship with both $C_{n T}$ and $C_{n}$, i.e., the widths of the correlation peaks in these coalescence variables depend on $1 / r_{T}$. This dependence is illustrated in Figs. 5 and 6 , in which the transverse radius is varied to show its effect in the transverse and radial correlation peaks. The longitudinal correlations are not shown because the longitudinal correlation peak widths are independent of $r_{T}$.

Fig. 5 shows the charged 2, 3, and 4 particle correlation distributions $R_{\sim}^{[t]}$ as functions of the transverse coalescence $C_{n T}$ over a range of values of $r_{T}$, the transverse radius of the source. The tixed parameters for these calculated functions are $y_{0}=p_{0}=\delta m_{\iota}=\delta y=0, \epsilon=1, m_{0}=3 \mu_{\pi}, \tau_{0}=4 \mathrm{fm} / \mathrm{c}$, and $\mathrm{T}=185.5 \mathrm{MeV}$. The transverse coalescence was varied over $0 \leq C_{n} T \leq 1.8$. The separate curves correspond to transverse radius value of $r_{T}=1$ (highest curve), $2,4,8,16$, and $32 \mathrm{fm}$ (lowest curve).

Fig. 6 shows the charged 2, 3, and 4 particle correlation distributions $R_{n}^{[ \pm]}$ as functions of the radial coalescence $C_{n R}$ over a range of values of $r_{T}$, the transrorse radius of the source. The fixed parameters for these calculated functions are $y_{0}=\phi_{0}=\delta m=\delta y=0, \epsilon=1, m_{0}=3 \mu_{\pi}, \tau_{0}=4 \mathrm{fm} / \mathrm{c}$, and $\mathrm{T}=185.5 \mathrm{MeV}$. The radial coalescence was varied over $0 \leq C_{n R} \leq 0.4$. The separate curi ss correspond to transverse radius value of $r_{T}=1$ (highest curve), $2,4,8,16$, and $32 \mathrm{fm}$ (lowest curve).

We note in Figs. 5 and 6 that while the peaking of the correlatio: distributions is very strong when the source radius is small, it becomes progressively weaker for larger radii. This is because the peak of the correlation distribution is forced into the smali coalescence region which is dominated by strong Coulomb repulsion. The extractiun of transverse source radii with charged pion Hanbury-Brown-Twiss correlations therefore will not be possible when the transverse radius of the emitting source approaches a value of $r_{r} \approx 32 \mathrm{fm}$. 


\section{DEPENDENCE ON TIME CONSTANT $\tau_{0}$}

Because the modified Bessel function $K_{0}(z)$ has the asymptotic behavior of an exponential in $z^{2}$, Equations (15) ar d (19) imply that the function $G\left(\vec{P}_{1}, \vec{P}_{2}\right)$ contains a term which is a Gaussian exponential with an exponent of the form $-2\left[\left(\mu_{\pi} / \hbar\right) \tau_{0} C_{2 L}\right]^{2}$ and a similar term involving $\left(m_{1}-m_{2}\right)$ which is related to $C_{2 R}$. This means that the emission time constant $\tau_{0}$ has a complementary relationship with $C_{n L}$ and possibly also with $C_{n R}$. This dependence is illustrated in Figs. 7 and 8 , in which the transverse radius is varied to show its effect in the longitudinal and radial correlation peaks. The transverse correlations are not shown because its correlation peak width is independent of $\tau_{0}$.

Fig. 7 shows the charged 2,3 , and 4 particle correlation distributions $R_{n}^{[ \pm]}$as functions of the longitudinai coalescence $C_{n L}$ over a range of values of $\tau_{0}$, the emission time constant of the source. The fixed parameters for these calculated functions are $y_{0}=\phi_{0}=\delta m=\delta \phi=0, \epsilon=1, m_{0}=3 \mu_{\pi}, r_{T}=10 \mathrm{fm}$, and $\mathrm{T}=185.5 \mathrm{MeV}$. The longitudinal coalescence was varied over $0 \leq C_{n L} \leq 1.8$. The separate curves correspond to source duration values of $\tau_{0}=1$ (highest curve), 2, 4, 8, 16, and $32 \mathrm{fm} / \mathrm{c}$ (lowest curve).

Fig. 8 shows the charged 2,3 , and 4 particle correlation distributions $R_{n}^{[ \pm]}$ as functions of the radial coalescence $C_{n R}$ over a range of values of $\tau_{0}$, the emission time constant of the source. The fixed parameters for these calculated functions are $y_{0}=\phi_{0}=\delta m=\delta \phi=0, \epsilon=1, m_{0}=3 \mu_{\pi}, r_{T}=10 \mathrm{fm}$, and $\mathrm{T}=185.5 \mathrm{MeV}$. The radial coalescence was varied over $0 \leq C_{n R} \leq 0.4$. The separate curves correspond to source duration values of $\tau_{0}=1$ (highest curve), 2, 4, 8, 16, and $32 \mathrm{fm} / \mathrm{c}$ (lowest curve).

Figs. 7 and 8 show a problem in the extraction of the source emission time constant which is similar to than encountered in extracting the source radius but less severe. When the transverse radius is about $r_{T} \approx 10 \mathrm{fm}$, the correlation peak disappears in the longitudinal correlation (Fig. 7) but persists in the radial correlation 
(Fig. 8). This rather unexpected result could permit extraction of $\tau_{0}$ in systems with moderate transverse radii but very large emission time constants, if such sources exist.

\section{DEPENDENCE ON PSEUDO-TEMPERATURE $T$}

Equations (15) and (19) also imply that $T$, the pseudo-temperature of the source will have some effect on both the longitudinal and radial correlations. This dependence is illustrated in Figs. 9 and 10, in which the pseudo-temperature is varied by a factor of four to show its effect on the longitudinal and radial correlation peaks. The transverse correlations are not shown because, in the Kolehmainen-Gyulassy model, the correlation peak width is independent of temperature.

Fig. 9 shows the charged 2, 3, and 4 particle correlation distributions $R_{n}^{[ \pm]}$ as functions of the longitudinal coalescence $C_{n L}$ over a range of values of $T$, the pseudo-temperature of the source. The fixed parameters for these calculated functions are $y_{0}=\phi_{0}=\delta m=\delta \phi=0, \epsilon=1, m_{0}=3 \mu_{\pi}, r_{T}=10 \mathrm{fm}$, and $\tau_{0}=4 \mathrm{fm} / \mathrm{c}$. The longitudinal coalescence was varied over $0 \leq C_{n L} \leq 1.8$. The separate curves correspond to pseudotemperature values of $T=186.1$ (highest curve), 268.4, 384.9, 549.5 , and $782.6 \mathrm{MeV}$ (lowest curve), which correspond to physical temperatures of $U=\mu_{\pi} \times 2^{n / 2}$ with $\mathrm{n}=0$ to 4 .

Fig. 10 shows the charged 2, 3, and 4 particle correlation distributions $R_{n}^{[ \pm]}$ as functions of the radial coalescence $C_{n R}$ over a range of values of $T$, the pseudotemperature of the source. The fixed parameters for these calculated functions are $y_{0}=\phi_{0}=\delta m=\delta \phi=0, \epsilon=1, m_{0}=3 \mu_{\pi}, r_{T}=10 \mathrm{fm}$, and $\tau_{0}=4 \mathrm{fm} / \mathrm{c}$. The radial coalescence was varied over $0 \leq C_{n R} \leq 1.8$. The separate curves correspond to pseudo-temperature values of $T=186.1$ (highest curve), 268.4, 384.9, 549.5, and 782.6 MeV (lowest curve), which correspond to physical temperatures of $U=\mu_{\pi} \times 2^{n / 2}$ with $\mathrm{n}=0$ to 4 .

It is interesting to note in this comparison that elevating the temperature, 
which shifts the pion momentum spectrum to higher energies, also has the effect of reducing both the longitudinal and the radial correlation peaks.

\section{CONCLUSION}

We have introduced a new set of Lorentz-invariant kinematic variables, the longitudinal, transverse, and radial coalescence, for describing the relative state of a group of pi mesons that are analysed with an n-particle correlation distribution $R_{n}$. The value of these variables has been illustrated in the examples above, it can be summarized as follows:

- The coalescence variables are dimensionless, Lorentz-invariant, monotone increasing functions of the more familiar relative rapidity $y$, transverse angle $\phi$, and transverse mass $m_{T}$ of the correlated pions.

- The coalescence variables allow direct comparisons among the correlation distributions for any number of pions.

- The coalescence variables for a multiparticle system are formed by adding the corresponding two-particle coalescence variables in quadrature for all particle pairs present in the system.

- The coalescence variables provide a Lorentz-invariant separation of the timelike and spacelike characteristics of the source of particles.

We have extended the analytic Kolehmainen-Gyulassy model by including the effects of source coherence asnd of Coulomb repulsion between the correlated pions. For the correlation distributions of order $n$ using the Kolehmainen-Gyulassy model, the longitudinal coalescence $C_{n L}$ has a complementary relation with the source duration $\tau_{0}$, the transverse coalescence $C_{n T}$ has a complementary relation with the 
source radius $r_{T}$, and the radial coalescence $C_{n R}$ has a complementary relation with a combination of $\tau_{0}$ and $r_{T}$. The results of this investigation can be summarized as follows:

- For source sizes greater than about $r_{T} \approx 32 \mathrm{fm}$, the two, three, and four particle correlation distributions peak in a region of coalescence where they are completely suppressed by Coulomb repulsion, making correlations of charged pions in this size domain insensitive to source size.

- It is apparently possible to extract emission time constants with values $\tau_{0} \approx$ $32 \mathrm{fm}$ by analyzing the correlation dependence on radial coalescence, provided the transverse radius of the source is of moderate size.

The author wishes to thank Profs. Geoffrey I. Opat, Lawrence Wilets, and Thomas A. Trainor for useful discussions and suggestions. This work was supported in part by the Division of Nuclear Sciences of the U. S. Department of Energy. 


\section{References}

${ }^{1}$ W. J. Willis, Nucl. Phys. A $418,425 \mathrm{c}(1984)$.

${ }^{2}$ W. A. Zajc, Phys. Rev. D 35, 3396 (1987).

${ }^{3}$ G. Goldhaber, Lawrence Berkeley Laboratory Report LBL-13291, (1981, unpublished).

${ }^{4}$ Y. M. Liu, D. Beavis, S. Y. Chu, S. Y. Fung, D. Keane, G. Van Galen, and M. Vient, Phys. Rev. C 34, 1667 (1986).

${ }^{5}$ W. A. Zajc, in Hadronic Multiparticle Production, edited by P. Carruthers, p. 125, (World Scientific, Singapore, 1987).

${ }^{6}$ David H. Boal, Claus-Konrad Gelbke, and Byron K. Jennings, Reviews of Modern Physics 62, 553 (1990).

${ }^{7}$ Karen Kolehmainen, Nucl. Phys. A $461,239 \mathrm{c}(1987)$.

${ }^{8}$ Karen Kolehmainen and Miklos Gyulassy, Phys. Lett. Bi80, 203 (1986).

${ }^{9}$ Larry McLerran, Rev. Mod. Phys. 58, 1021 (1986).

${ }^{10}$ M. Gyulassy, S. K. Kauffmann, and Lance W. Wilson, Phys. Rev. C 20, 2267 (1979).

11 M. Biyajima, A. Bartl, T. Mizoguchi, O. Terazawa, and N. Suzuki, Preprint UWThPh-1990-3 (March 5, 1990, unpublished). 


\section{Figure Captions}

FIG. 1. The coalescencr varisbles $C_{n L}, C_{n T}$, and $C_{n R}$ for $\mathrm{n}=2$ (solid curves), $\mathrm{n}=3$ (dashed curves), and $\mathrm{n}=4$ (dot-dash curves) are plotted against the kinematic difference parameters $\delta \mathrm{y}, \delta \phi$, and $\delta \mathrm{m}$ for $y_{0}=\phi_{0}=0$, and $m_{0}=3 \mu_{\pi}$.

FIG. 2. Comparison of 2, 3, and 4 particle correlation distributions for neutral particlec $k_{n}^{[0]}$ (dashed curves) and for charged particles $R_{n}^{[ \pm]}$(solid curves) as functions of the longitudinal coalescence $C_{n L}$. The parameters used are given in the text.

FIG. 3. Comparison of 2, 3, and 4 particle correlation distributions for neutral particles $R_{n}^{[0]}$ (dashed curves) and for charged particles $R_{n}^{[t]}$ (solid curves) as functions of the transverse coalescence $C_{n T}$. The parameters used are given in the text.

FIG. 4. Comparison of 2, 3, and 4 particle correlation distributions for neutral particles $R_{n}^{[0]}$ (dashed curves) and for charged particles $R_{n}^{[t]}$ (solid curves) as functions of the radial coalescence $C_{n R}$. The parameters used are given in the text.

FIG. 5. The 2, 3, and 4 charged particle correlation distributions $R_{n}^{[ \pm]}$as functions of the transverse coalescence $C_{n T}$ over a range of values of $r_{T}$, the transverse radius of the source. The parameters used are given in the text. The separate curves correspond to transverse radii of $r_{T}=1$ (highest curve), $2,4,8,16$, and $32 \mathrm{fm}$ (lowest curve).

FIG. 6. The 2, 3, and 4 charged particle correlation distributions $R_{n}^{[ \pm]}$as functions of the radial coalescence $C_{n R}$ over a range of values of $r_{T}$, the transverse radius of the source. The 
parameters used are given $i$ in the text. The separate curves correspond to transverse radii of $r_{T}=1$ (highest curve), 2, 4, 8, 16, and $32 \mathrm{fm}$ (lowest curve).

FIG. 7. The 2, 3, and 4 charged particle correlation distributions $R_{n}^{[ \pm]}$as functions of the lougitudinal coalescence $C_{n L}$ over a range of values of $\tau_{0}$, the emission time constant of the source. The parameters used are given in the text. The separate curves correspond to source emission time constants of $\tau_{0}=1$ (highest curve), 2, 4, 8, 16, and $32 \mathrm{fm} / \mathrm{c}$ (lowest curve).

FIG. 8. The 2, 3, and 4 charged particle correlation distributions $R_{n}^{[ \pm]}$as functions of the radial coalescence $C_{n R}$ over a range of values of $\tau_{0}$, the emission time constant of the source. The parameters used are given in the text. The separate curves correspond to source emission time constants of $\tau_{0}=1$ (highest curve), 2, 4, 8, 16, and $32 \mathrm{fm} / \mathrm{c}$ (lowest curve).

FIG. 9. The charged 2, 3, and 4 particle correlation distributions $R_{n}^{[t]}$ as functions of the longitudinal coalescence $C_{n L}$ over a range of values of $T$, the pseudo-temperature of the source. The parameters used are given in the text. The separate curves correspond to pseudo-temperature values of $T=186.1$ (highest curve), 268.4, 384.9, 549.5 , and $782.6 \mathrm{MeV}$ (lowest curve), which correspond to physical temperatures of 4 .

FIG. 10. The charged 2, 3, and 4 particle correlation distributions $R_{n}^{[ \pm]}$as functions of the radial coalescence $C_{n R}$ over a range of values of $T$, the pseudo-temperature of the source. The parameters used are given in the text. The separate curves correspond to pseudotemperature values of $T=186.1$ (highest curve), 268.4,384.9, 549.5, and $782.6 \mathrm{MeV}$ (lowest curve), which correspond to physical temperatures of $U=\mu_{\pi} \times 2^{n / 2}$ with $n=0$ to 4 . 

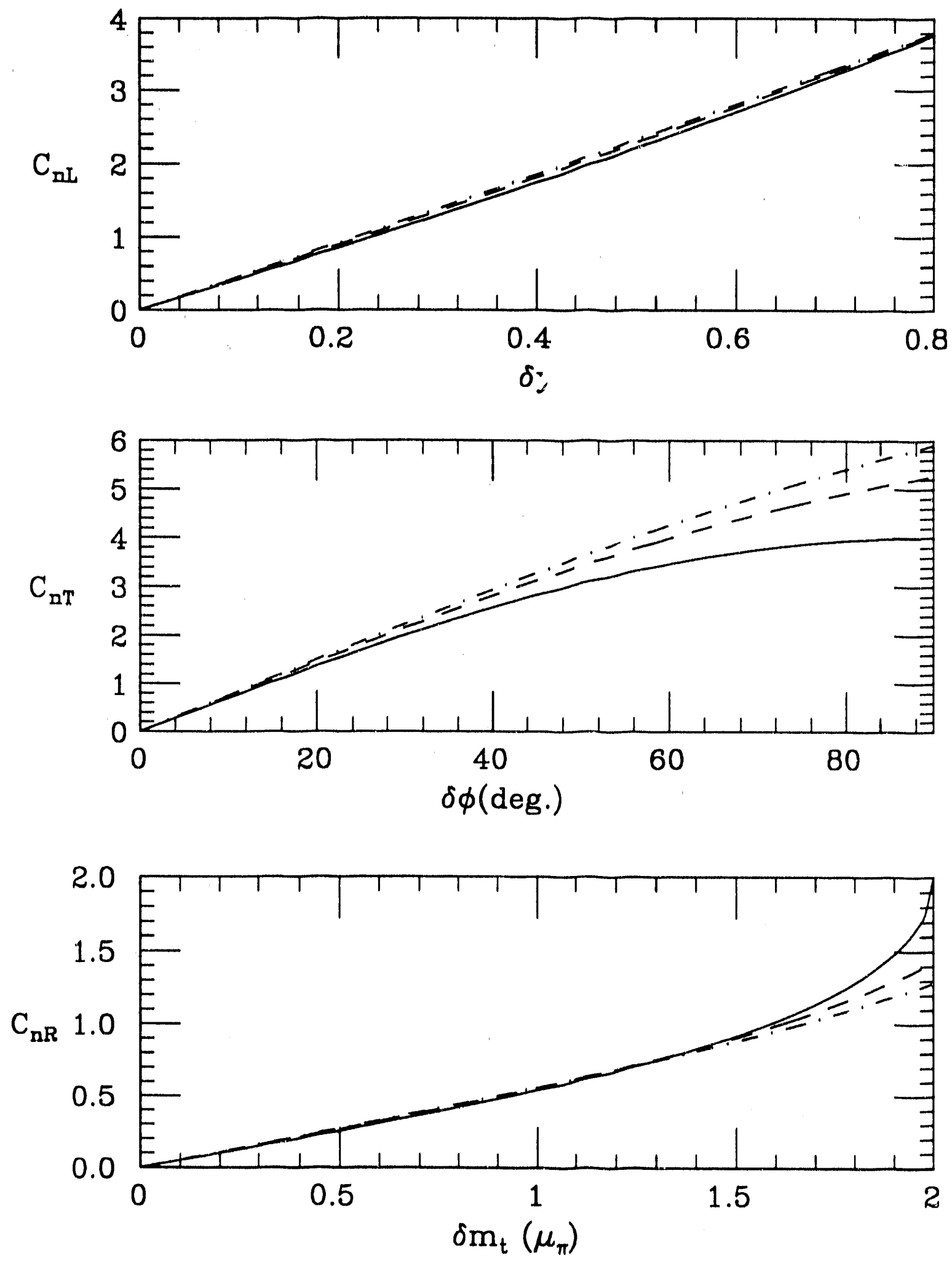

Fig. 1 


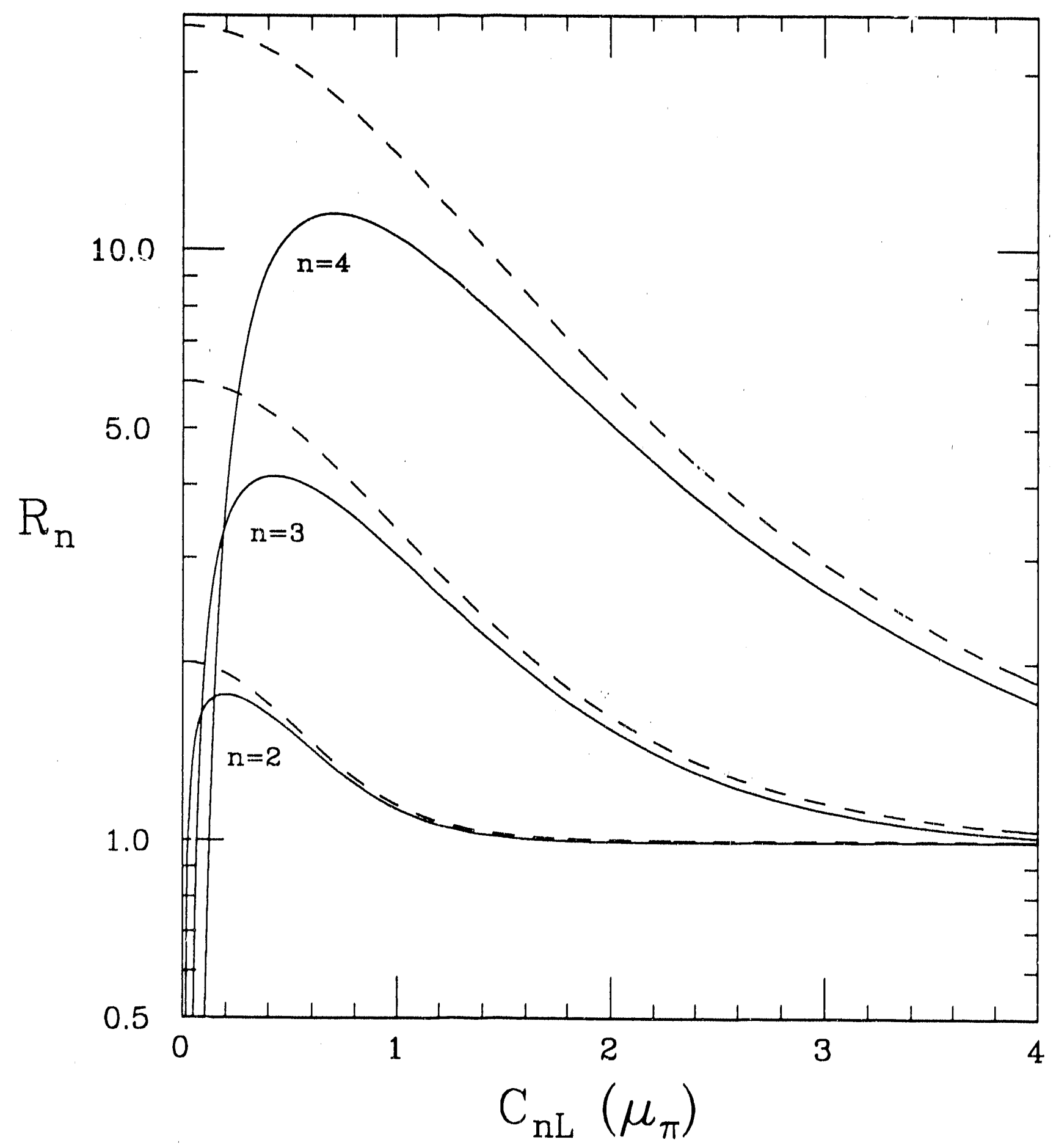

Fig. 2 


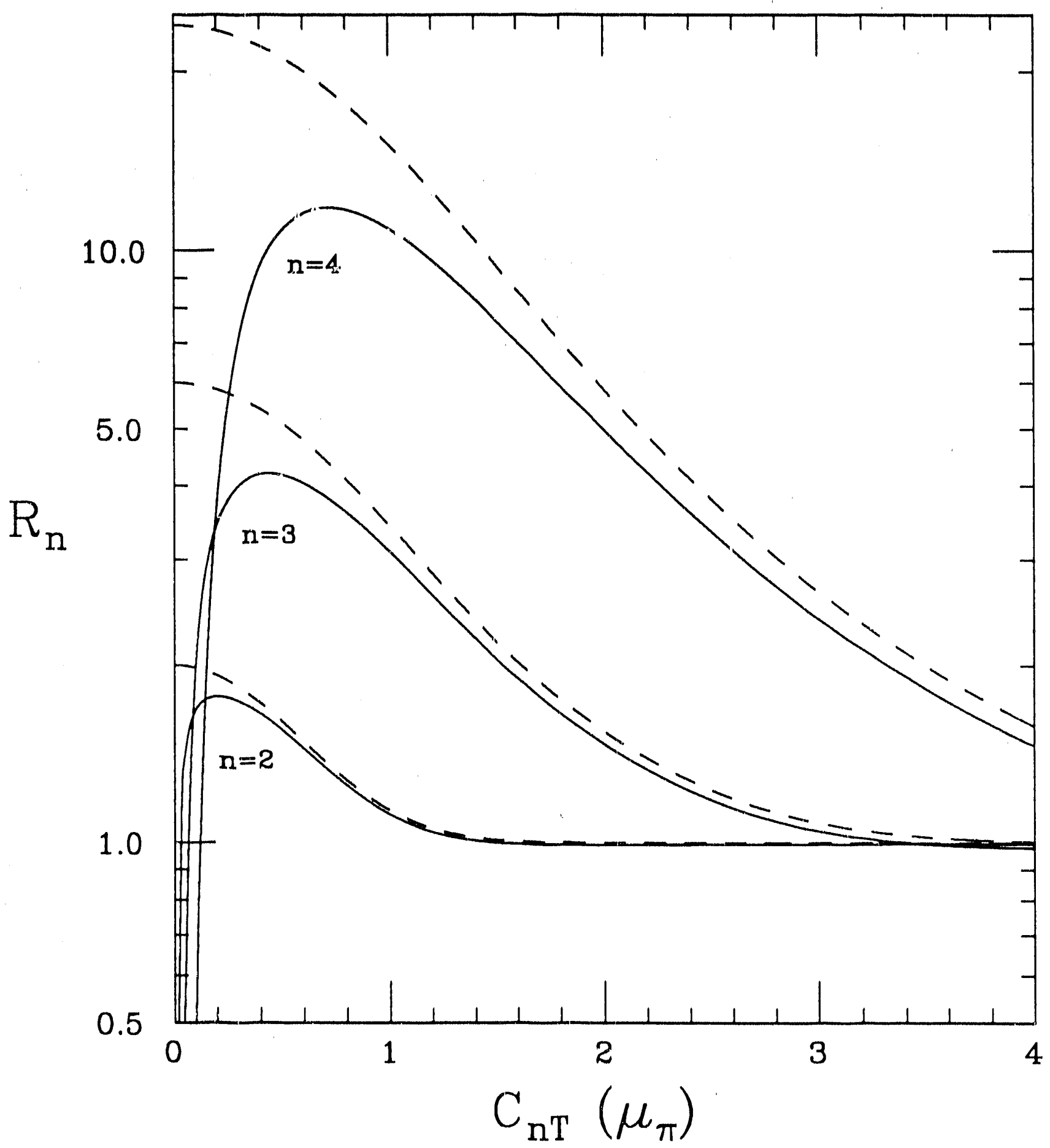

Fig. 3 


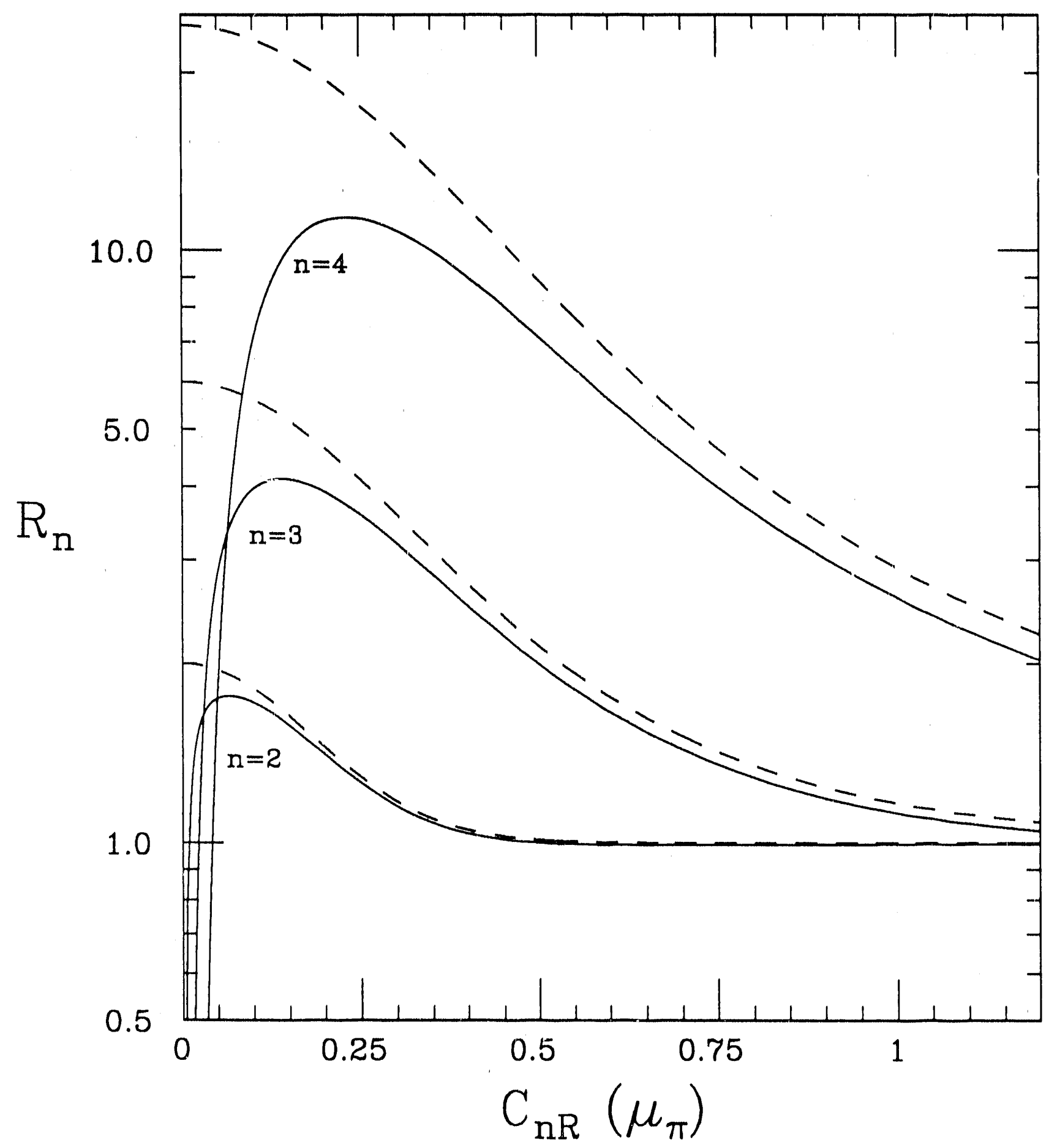

Fig. 4 

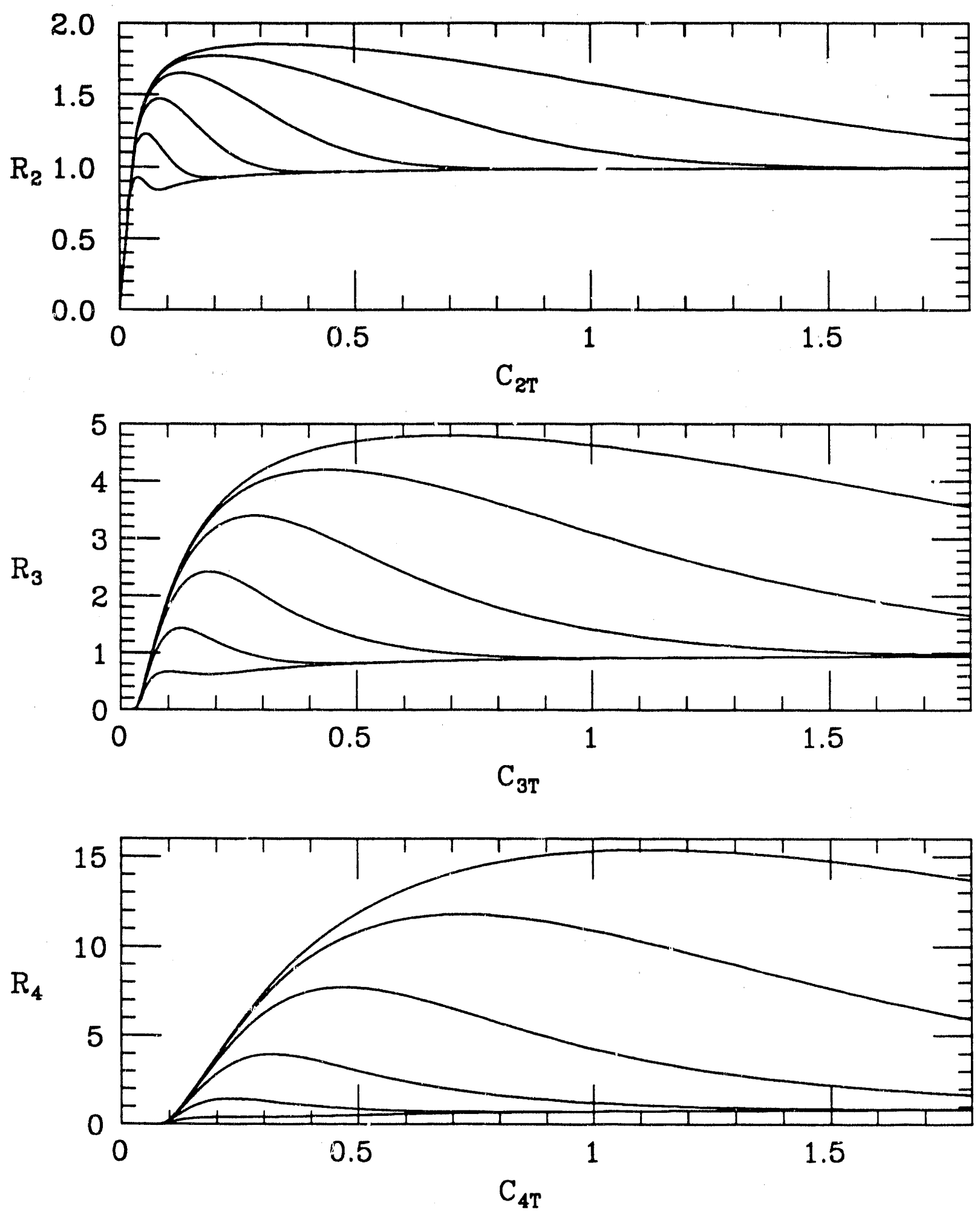

Fig. 5 

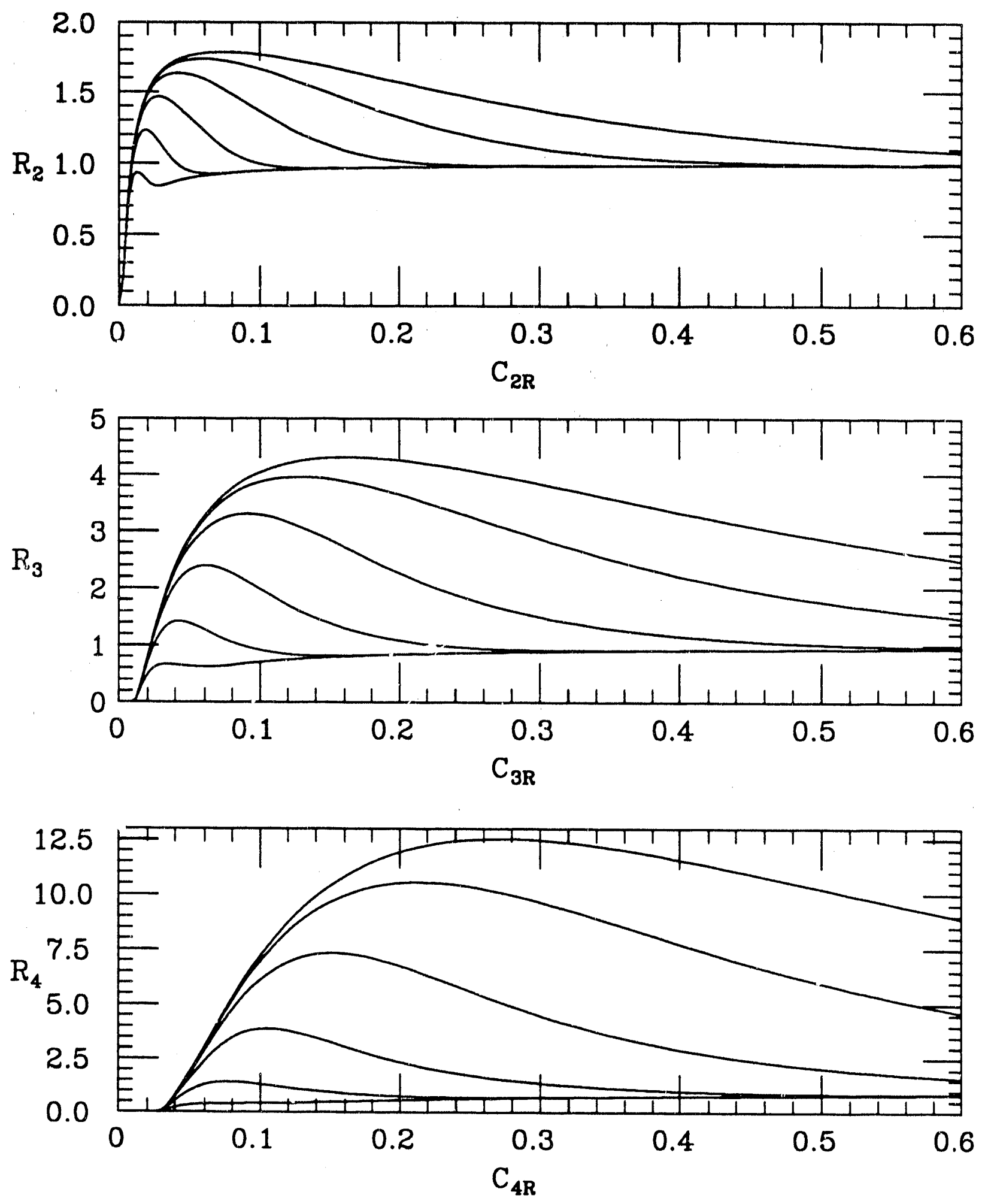

Fig. 6 

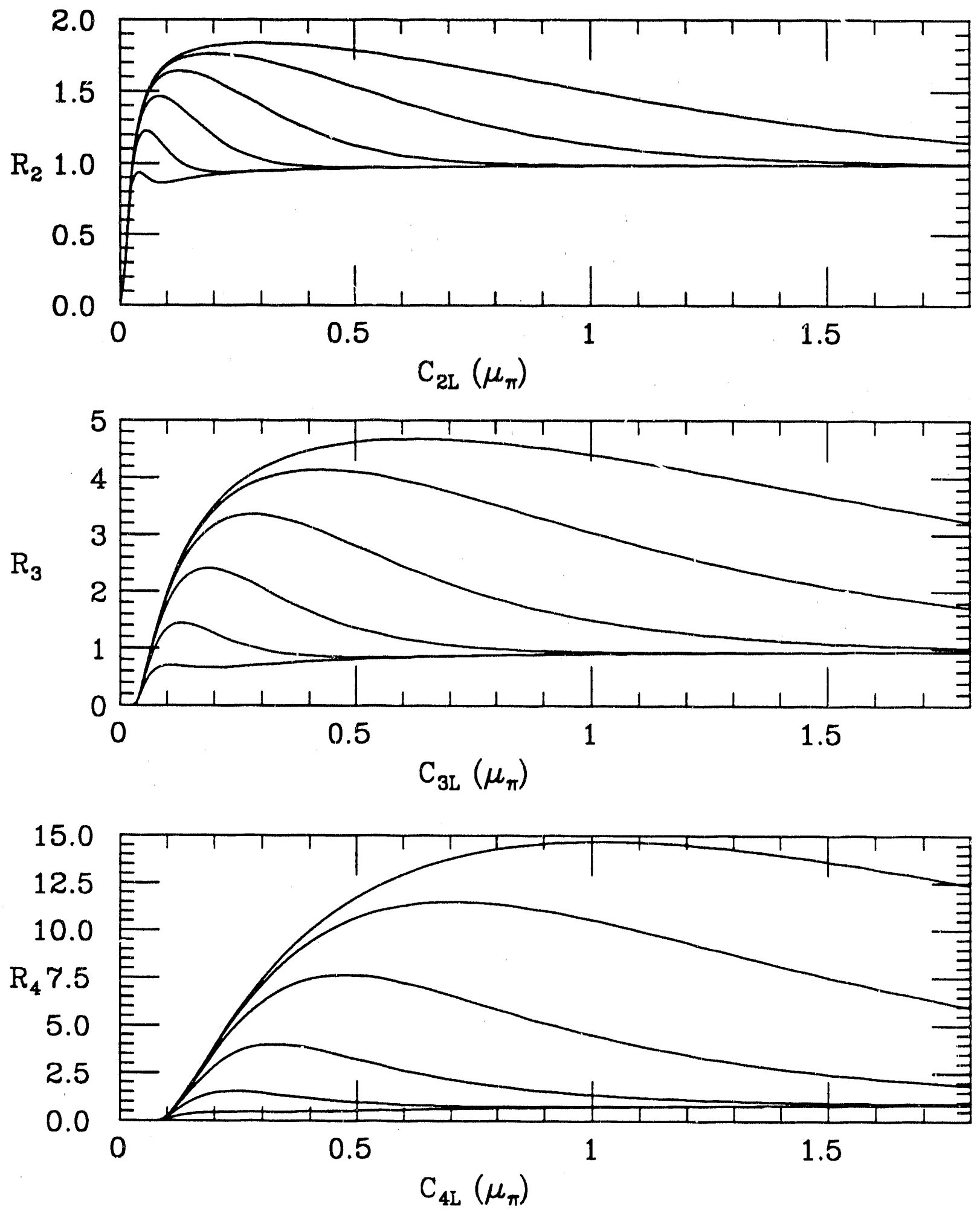

Fig. 7 

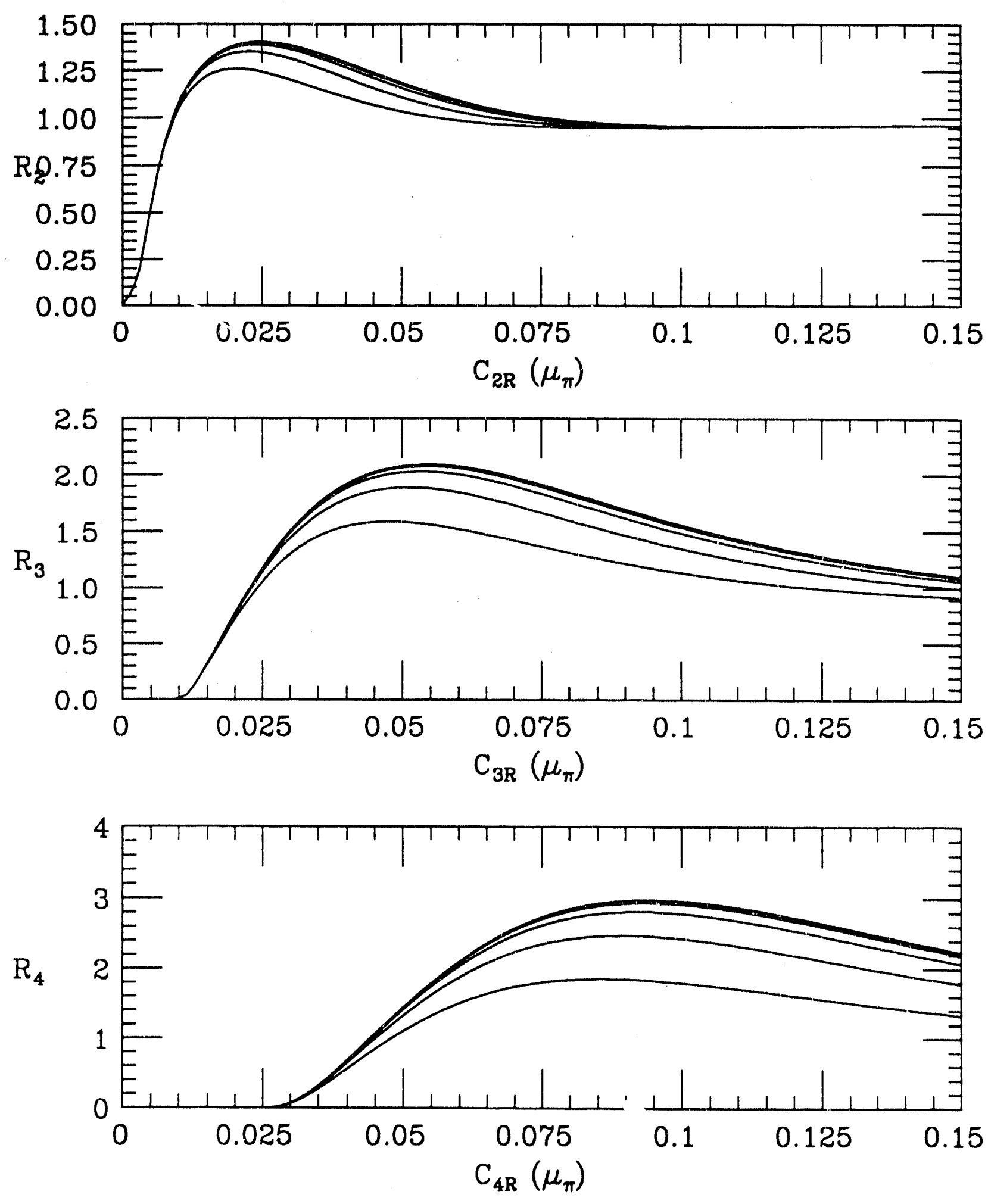

Fig. 8 

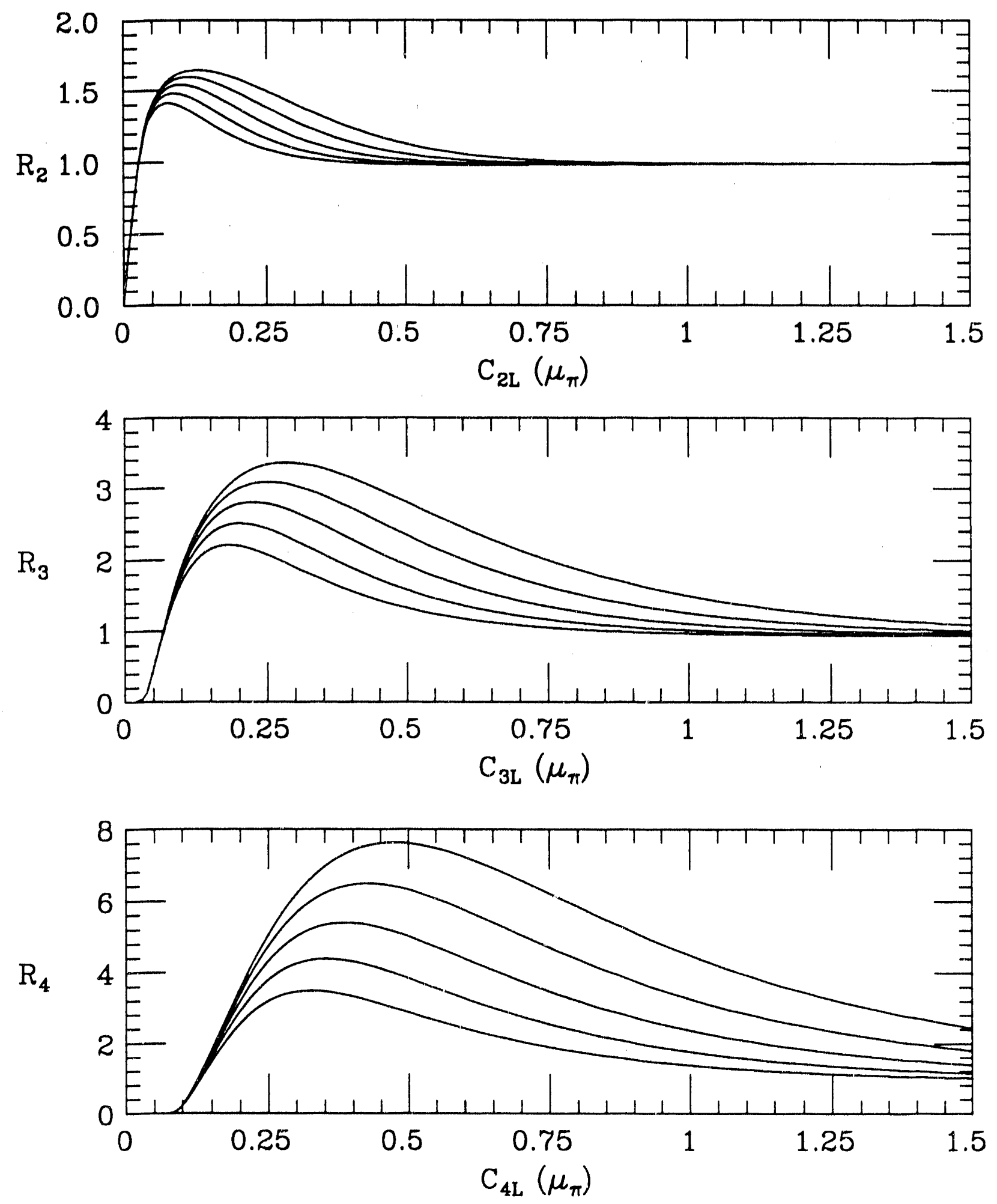

Fig. 9 

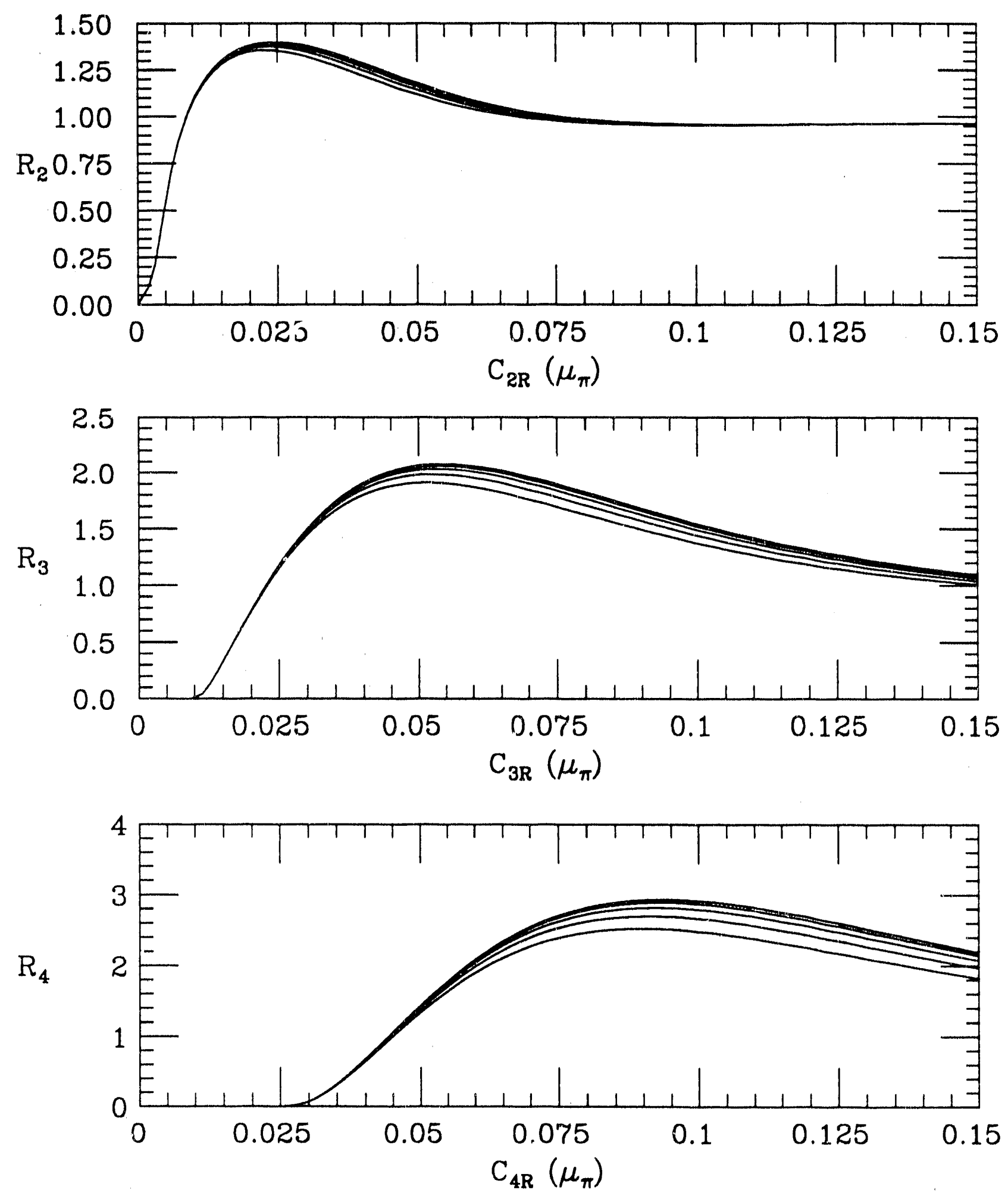

Fig. 10 

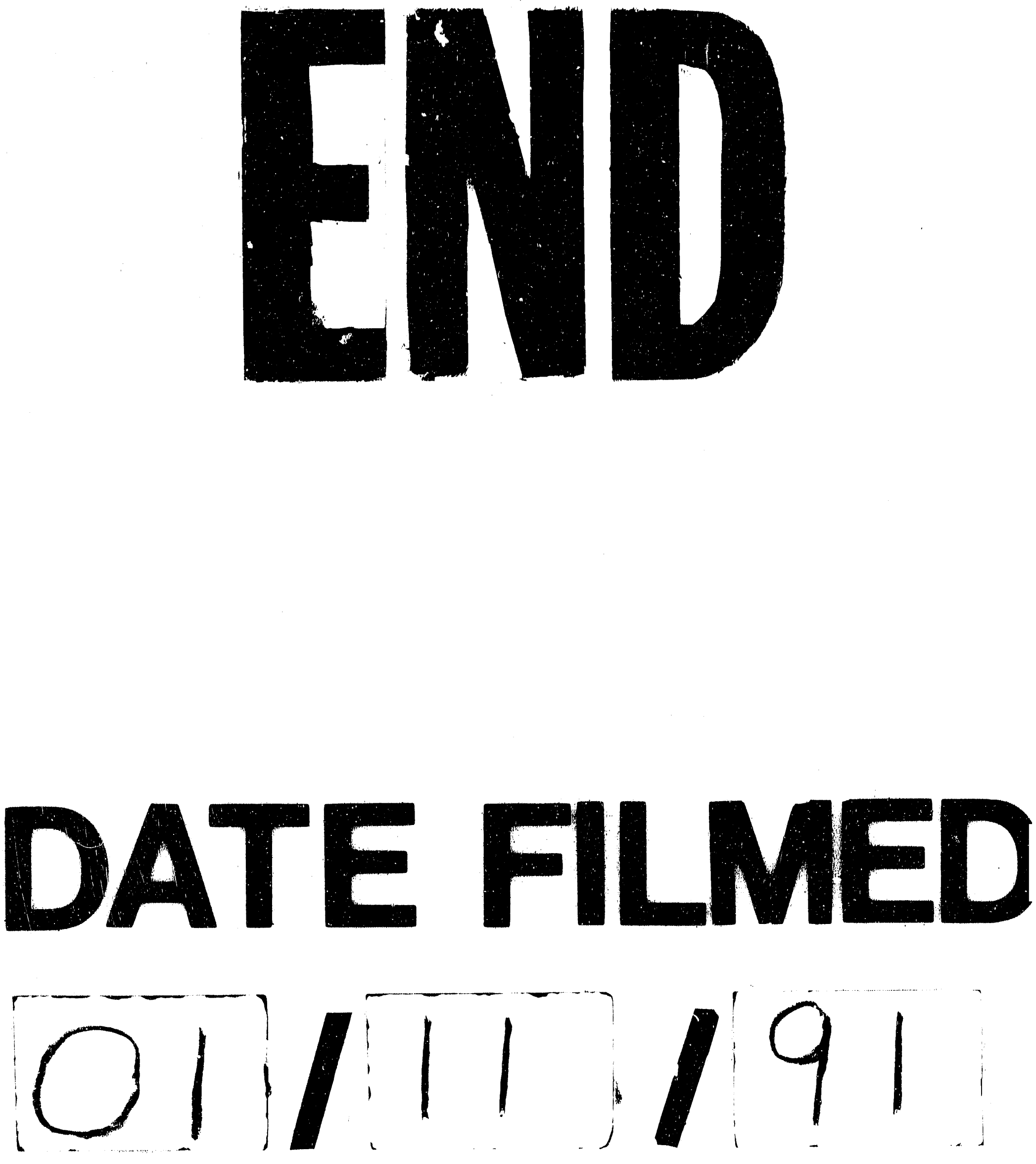
